\title{
Does Subcutaneous Infiltration of Liposomal Bupivacaine Following Single-Level Transforaminal Lumbar Interbody Fusion Surgery Improve Immediate Postoperative Pain Control?
}

\author{
Marko Tomov ${ }^{1}$, Kevin Tou ${ }^{2}$, Rose Winkel ${ }^{2}$, Ross Puffer ${ }^{3}$, Mohamad Bydon $^{3}$, Ahmad Nassr ${ }^{1}$, \\ Paul Huddleston ${ }^{1}$, Michael Yaszemski ${ }^{1}$, Bradford Currier ${ }^{1}$, Brett Freedman ${ }^{1}$ \\ ${ }^{1}$ Department of Orthopedic Surgery, Mayo Clinic, Rochester, MN, USA \\ ${ }^{2}$ Department of Orthopedic Surgery, United States Army, Landstuhl, Germany \\ ${ }^{3}$ Department of Neurosurgery, Mayo Clinic, Rochester, MN, USA
}

\section{Study Design: Retrospective case-control study using prospectively collected data.}

Purpose: Evaluate the impact of liposomal bupivacaine (LB) on postoperative pain management and narcotic use following standardized single-level low lumbar transforaminal lumbar interbody fusion (TLIF).

Overview of Literature: Poor pain control after surgery has been linked with decreased pain satisfaction and increased economic burden. Unfortunately, opioids have many limitations and side effects despite being the primary treatment of postoperative pain. LB may be a form of pre-emptive analgesia used to reduce the use of postoperative narcotics as evidence in other studies evaluating its use in single-level microdiskectomies.

Methods: The infiltration of LB subcutaneously during wound closure was performed by a single surgeon beginning in July 2014 for all single-level lumbar TLIF spinal surgeries at Landstuhl Regional Medical Center. This cohort was compared against a control cohort of patients who underwent the same surgery by the same surgeon in the preceding 6 months. Statistical analysis was performed on relevant variables including: morphine equivalents of narcotic medication used (primary outcome), length of hospitalization, Visual Analog Scale pain scores, and total time spent on a patient-controlled analgesia (PCA) pump.

Results: A total of 30 patients were included in this study; 16 were in the intervention cohort and 14 were in the control cohort. The morphine equivalents of intravenous narcotic use postoperatively were significantly less in the LB cohort from day of surgery to postoperative day 3. Although the differences lost their statistical significance, the trend remained for total (oral and intravenous) narcotic consumption to be lower in the LB group. The patients who received the study intervention required an acute pain service consult less frequently (62.5\% in LB cohort vs. $78.6 \%$ in control cohort). The amount of time spent on a PCA pump in the LB group was 31 hours versus 47 hours in the control group $(p=0.1506)$.

Conclusions: Local infiltration of LB postoperatively to the subcutaneous tissues during closure following TLIF significantly decreased the amount of intravenous narcotic medication required by patients. Well-powered prospective studies are still needed to determine optimal dosing and confirm benefits of LB on total narcotic consumption and other measures of pain control following major spinal surgery.

Keywords: Lumbar; Lumbar interbody fusion; Polyetheretherketone cages; Chronic pain

Received Apr 12, 2017; Revised May 8, 2017; Accepted May 31, 2017

Corresponding author: Marko Tomov

Department of Orthopedic Surgery, Mayo Clinic, 200 First Street SW, Rochester, MN 55905, USA

Tel: +1-507-284-2511, E-mail: tomov.marko@mayo.edu 


\section{Introduction}

Despite advancements in pain management modalities, acute pain control following major spinal surgery continues to be a challenge [1]. Poor pain control following surgery has been linked with decreased patient satisfaction and increased economic burden due to delays in discharge [2]. Medical benefits of optimized postoperative pain control range from an improvement in cardiac, respiratory and gastrointestinal functions, to a reduced mortality [2]. Opioids have been the primary treatment for acute pain following spine surgery; however, there are limitations and side effects associated with their use. These include central nervous system and respiratory depression, ileus, nausea, vomiting, itching, and development of hyperalgesia [2]. Furthermore, opioid dependence and abuse has become an area of major concern and criticism of the healthcare system of the United States [3].

Due to the limitations of opioid pain medications, alternatives to and mitigation strategies for opioid dominant pain control approaches are being investigated to help manage pain in the acute setting following surgery [4]. One of these modalities is pre-emptive analgesia, via the infiltration of local anesthetic into the surgical site. Bupivacaine $\mathrm{HCl}$ is one of the longest acting local anesthetics and is widely used for neurological blockade. Despite extended duration of effect over lidocaine and other local anesthetics, its duration of action is still relatively short compared to the typical period of maximal postoperative pain following major spinal surgery [5]. To overcome this limitation, liposomal bupivacaine (LB) was recently approved for administration into the surgical site to produce postoperative analgesia. Its commercial name is Exparel. LB is released over a 72-hour period using Depofoam as a delivery platform, a microscopic lipid based structure which encapsulates the medication and erodes over time to release the anesthetic [6]. The use of LB has been studied in several orthopedic procedures including total knee, hip and shoulder arthroplasties, and orthopedic trauma cases, as well as in the fields of general surgery, plastic surgery, and colorectal surgery [7-16]. However, studies for its utility in spine surgery have been limited $[3,4,17]$.

Studies that have assessed the use of LB in spine surgery show promising results. In single-level microdiskectomies, it was found that use of intravenous narcotics was significantly decreased following infiltration of LB into the subcutaneous tissues during closure [4]. However, compara- tive effectiveness of LB infiltration versus a well-matched, control cohort following larger spine procedures, such as interbody fusions, is lacking. The purpose of this study was to evaluate the impact of LB on postoperative pain management and narcotic use following a standardized single-level transforaminal lumbar interbody fusion (TLIF).

\section{Materials and Methods}

A pre-requisite set by our pharmacy and therapeutics committee for addition of LB (Exparel; Pacira Pharmaceuticals Inc., Parsippany, NJ, USA) to our formulary, was empiric demonstration that the efficacy of LB was superior or equal to our current standard of care practice, which included no postoperative local anesthetic infiltration. Thus, a 6-month process improvement (PI) project was proposed and approved by our quality assurance and human protections administrator.

Under this PI project the immediate clinical outcomes as they relate to pain control and the demographics of consecutive patients under-going single-level TLIF by a single surgeon applying a standardized surgical technique who received a subcutaneous LB field block at the end of the procedure were collected and compared to a historical cohort of consecutive TLIF cases that had been completed in the 6-months prior to the initial use of LB at our facility (July 2014) by the same surgeon performing the same surgical technique. To identify the comparator cohort, the electronic surgical scheduling system was queried for all single-level TLIF procedures performed in the 6 months prior to the initial use of LB at our facility. The prospectively maintained electronic medical record was queried and abstracted for all data from both cohorts analyzed. Outcome measures were selected to reflect clinically meaningful endpoints related to acute pain control and for the reliability of their reporting in the electronic medical record. Accurate documentation of the administration of narcotic pain medications is one of the most regulated and reviewed actions in the medical record, thereby limiting the impact of recall bias on the retrospectively abstracted data. This project analyzed the 30 patients who underwent elective single-level TLIF for degenerative spinal conditions that failed to respond to an appropriate course of nonoperative therapy by one fellowship trained spine surgeon at one military medical center between January 2014 to December 2014. Nonoperative therapy in 
all patients included at least the following measures: activity modification, nonsteroidal anti-inflammatory drugs, a lumbar stabilization physical therapy regimen, and selective epidural and/or facet injections under the direction of fellowship trained pain physicians.

To evaluate the impact of LB on postoperative pain management in patients undergoing a single level TLIF during the prospective phase of the PI project, LB was injected into the subcutaneous tissue in a cohort of 16 consecutive patients. This cohort was compared to a cohort of 14 consecutive patients who did not receive LB in the 6 months prior to the initiation of the PI project. Relevant variables were compared including: length of hospitalization, requirements for oral and intravenous narcotics in the postoperative period, Visual Analog Scale (VAS) for pain, and total time on intravenous narcotic pain medications.

The electronic medical record was used to gather demographic data from both cohorts in addition to information regarding pain medication use postoperatively. Some of these essential variables included average VAS scores at different time points both preoperatively and postoperatively, the morphine equivalents of oral and intravenous narcotic pain medications needed from day of surgery (DOS) to postoperative day (POD) 3, total time spent on a patient-controlled analgesia (PCA) pump, and percentage of patients requiring an acute pain service (APS) consult. POD 3 was selected as the cut-off date for assessment and data analysis, since it covers the expected duration of effect of LB and it's the last day in which all patients were still hospitalized following their spinal fusion surgery. The LB and control cohorts were compared using multiple independent Student $t$-test with a significance level set to $p<0.05$.

\section{Surgical procedure and postoperative care}

All patients in this study underwent a primary (i.e., no prior surgery at the surgical level) elective single level TLIF. Preoperative fluoroscopy was used to localize prior to incision. After the patient was prepped and draped, $10 \mathrm{~mL}$ of regular $0.5 \%$ bupivacaine with epinephrine was infiltrated intradermal and subcutaneously in the area of the planned incision in all cases in both cohorts. This was standard of care at our facility and was not a part of our LB intervention. After skin incision, the fascia was opened and paravertebral muscles were dissected subperiosteally using the using electrocautery. The TLIF-level was confirmed with fluoroscopy and the capsule was taken down at the TLIF level and preserved at the next cephalad level. Bilaterally, the cephalad and caudal transverse processes (or sacral ala) were then exposed to their tips and decorticated along with the lateral facets to create a posterolateral gutter for bone grafting. Self-retaining retractors were placed. A matchstick bur, osteotome, and/or Kerrison rongeurs were used to remove the inferior articulating process and lateral lamina of the cephalad level and the superior and medial articulating processes of the caudal level on the side of predominant leg symptoms. In all cases a single side was approached for the interbody portion of the procedure, while the contralateral side was preserved and the facet was decorticated and packed with bone graft. The dural tube was retracted medially to visualize the posterolateral corner of the disk. A disk knife was used to create a box annulotomy. Paddle dilators, paddle shaver, and serrated curettes were used to remove the nucleus pulposus and endplate cartilage to expose bleeding bony end plates above and below. Following irrigation of the disk space, loose milled autograft bone was injected into the anterior and contralateral portions of the disk space, an appropriately sized bullet-shaped cage (Medtronic Capstone, polyetheretherketone; Medtronic, Minneapolis, MN, USA) was inserted, and its correct positioning was confirmed with lateral fluoroscopy. After satisfactory placement of the cage, pedicle screws (Medtronic Legacy 5.5 Ti, Medtronic) were placed using a free-hand technique with lateral fluoroscopy assist. Rods and set screws were placed, compressed, and final tightened. The wound was closed in layers. Following water-tight closure of the fascia, the wound of the patients in the LB cohort was infiltrated with $40 \mathrm{~mL}$ of a 50:50 mixture of LB and 0.5\% bupivacaine without epinephrine; $20 \mathrm{~mL}$ of the mixture was injected on either side of midline with an 18-gauge spinal needle. All patients were adequately recovered in the postanesthesia care unit and transferred to the in-patient ward for acute convalescence and rehabilitation. All patients were routinely instructed during their preoperative counseling visit to expect to stay 5 days following surgery. The primary oral narcotic pain medication administered to patients in the postoperative period was oxycodone/ acetaminophen ( $5 \mathrm{mg} / 325 \mathrm{mg}$ ) every 4 hours as needed. Intravenous hydromorphone was given for breakthrough pain (2 mg every 2 hours) not responsive to the primary pain medications. PCA was not prescribed routinely, but 
Table 1. Demographics data for LB group versus control group

\begin{tabular}{lccc} 
Characteristic & LB group & Control group & $p$-value (two-tailed) \\
No. of subjects & 16 & 14 & - \\
Male & 16 & 12 & - \\
\hline Female & 0 & 39 & - \\
Average age $(\mathrm{yr})$ & 41 & $24-54$ & - \\
Age range $(\mathrm{yr})$ & $27-66$ & 28.7 & - \\
Average BMl $\left(\mathrm{kg} / \mathrm{m}^{2}\right)$ & 29 & $22.0-35.8$ & - \\
BMI range $\left(\mathrm{kg} / \mathrm{m}^{2}\right)$ & $21.1-33.5$ & 6.1 & - \\
\hline Preoperative average pain score & 5.9 & 47.1 & 0.7463 \\
\hline Average preoperative Oswestry Disability Index & 52.8 & 0.3392 \\
\hline
\end{tabular}

LB, liposomal bupivacaine; BMI, body mass index.

was typically prescribed in patients for which routine pain medications failed to provide sufficient relief and an APS consult had been obtained.

\section{Results}

There were a total of 30 patients in this study. Sixteen patients (53\%) underwent a single-level TLIF with infiltration of LB into the superficial portion of the wound prior to closure (LB cohort). A total of 14 patients (47\%) underwent a single-level TLIF without infiltration of LB at any point during the case and without administration of local anesthetic to the surgical site during the closure (control cohort). The complete demographics data is shown in Table 1; there were no significant differences in demographics data between the two groups, including gender distribution, age, BMI, preoperative average pain score, or average preoperative Oswestry Disability Index (ODI).

The most significant difference between the LB cohort and the control cohort was the amount of intravenous narcotic used on the DOS out to POD 3. The LB group used statistically significant less intravenous narcotic on DOS (LB group, 5.2 morphine equivalents versus control group, 23.7 morphine equivalents; $p=0.005$ ), POD 1 (LB group, 20.1 morphine equivalents versus control group, 58.4 morphine equivalents; $p=0.0175$ ), POD 2 (LB group, 9.7 morphine equivalents versus control group, 44.9 morphine equivalents; $p=0.001$ ), and POD 3 (LB group, 3.9 morphine equivalents versus control group, 21.4 morphine equivalents; $p=0.0002$ ). These differences translate into a 4.6-fold decrease on DOS, 2.9-fold decrease on POD 1, 4.6-fold decrease on POD 2, and 5.5-fold decrease

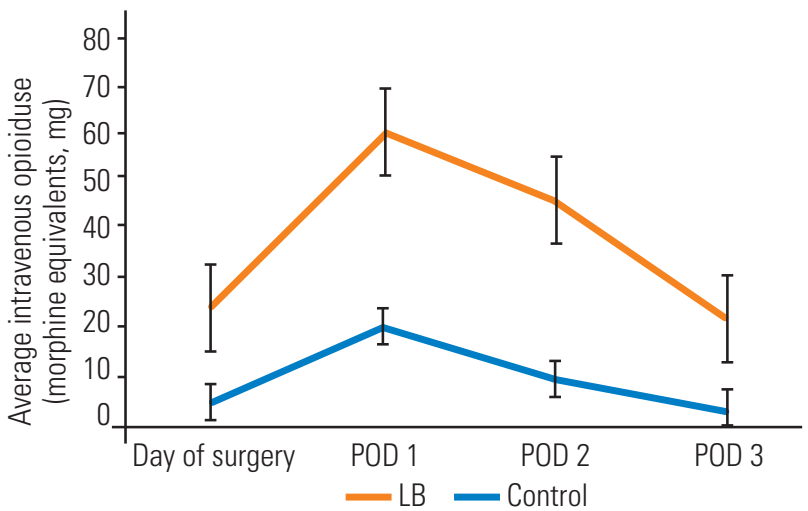

Fig. 1. Comparison of average intravenous opioid use for patients treated with LB group vs. control group. LB, liposomal bupivacaine; POD, postoperative day.

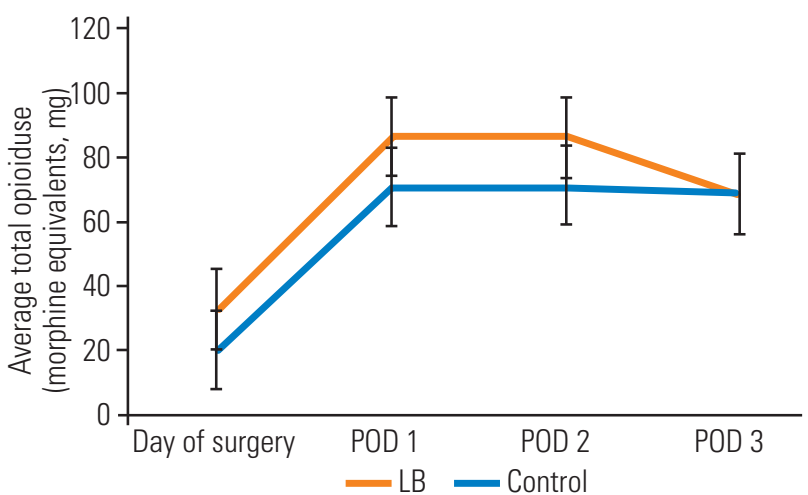

Fig. 2. Comparison of average total opioid use for patients treated with LB group vs. control group. LB, liposomal bupivacaine; POD, postoperative day.

on POD 3, of intravenous narcotic use, expressed graphically in Fig. 1. Although not statistically significant, there was a trend towards lower total (oral and intravenous) 
Table 2. Operative and pain measurements for LB group versus control group

\begin{tabular}{|c|c|c|c|}
\hline Variable & LB group & Control group & $p$-value (two-tailed) \\
\hline Average operative time & $4 \mathrm{hr} 9 \min$ & $5 \mathrm{hr} 1 \mathrm{~min}$ & 0.2520 \\
\hline Average length of stay (day) & 4.8 & 5.1 & 0.6020 \\
\hline$\%$ of patients with acute pain service consult & 62.5 & 78.6 & - \\
\hline DOS morphine equivalent of total narcotics (average) & 20.1 & 32.8 & 0.1913 \\
\hline DOS morphine equivalent of intravenous narcotics (average) & 5.2 & 23.7 & 0.0051 \\
\hline POD 1 morphine equivalent of total narcotics (average) & 70.9 & 86.3 & 0.5019 \\
\hline POD 1 morphine equivalent of intravenous narcotics (average) & 20.1 & 58.4 & 0.0175 \\
\hline POD 2 morphine equivalent of total narcotics (average) & 71.6 & 86.2 & 0.4734 \\
\hline POD 2 morphine equivalent of intravenous narcotics (average) & 9.7 & 44.9 & 0.001 \\
\hline POD 3 morphine equivalent of total narcotics (average) & 68.8 & 68.6 & 0.9902 \\
\hline POD 3 morphine equivalent of intravenous narcotics (average) & 3.9 & 21.4 & 0.0002 \\
\hline Average total time on patient controlled analgesia (hr) & 31.3 & 47.1 & 0.1506 \\
\hline DOS VAS (average) & 4.1 & 3.2 & 0.2428 \\
\hline POD 1 VAS (average) & 4.1 & 3.2 & 0.1893 \\
\hline POD 2 VAS (average) & 4.5 & 3.8 & 0.3648 \\
\hline POD 3 VAS (average) & 5.2 & 4.8 & 0.5358 \\
\hline 10-Day wound check VAS (average) & 4.5 & 4.2 & 0.7854 \\
\hline 6-Week follow-up VAS (average) & 2.8 & 3.5 & 0.4508 \\
\hline
\end{tabular}

LB, liposomal bupivacaine; DOS, day of surgery; POD, postoperative day; VAS, Visual Analog Scale.

narcotic consumption in the LB cohort with an eventual congruence between the two cohorts on POD 3 as visualized in Fig. 2. However, only $62.5 \%$ of the LB group patients required an APS consult for failure of the standard pain control regimen versus $78.6 \%$ in the control group. Despite a lower amount of time spent on a PCA pump in the LB group (31 hours) versus the control group (47 hours), this was not statistically significant $(p=0.1506)$. In addition, there was no statistical difference between the two cohorts for average number of days spent in the hospital, since both groups typically stayed to the predestined 5th day. Despite the LB cohort requesting significantly less intravenous narcotic pain medication, the average VAS for pain at any time point investigated was not significantly different, as demonstrated in Table 2.

\section{Discussion}

Pain control has become a priority for all hospitalizations. Pain management questions represent 3 of 27 questions asked in the Hospital Consumer Assessment of Healthcare Providers and Systems, which is the survey used by the Centers for Medicare and Medicaid to measure healthcare provider and systems performance. Essentially all validated patient-reported outcome measures for spine surgery have some pain domain. Postoperative pain control in spine surgery is challenging. Many patients undergoing spine surgery are chronic pain patients, which further renders postoperative pain management difficult. Therefore, modalities that can reduce pain and improve other process measures of care need to be assessed and when shown to be effective, these modalities should be implemented to improve healthcare outcomes and reduce costs.

Pre-emptive analgesia is a concept originating from the work of Woolf [18] in the early 1980's in which he hypothesized a mechanism by which tissue injury induces post-injury pain hypersensitivity and that pre-emptive blockade of this mechanism can limit this pain. In a follow-up review article in 1993 by Woolf and Chong [19], concepts of pre-emptive analgesia in surgical practice were further explored, ranging from different modalities of pain control to the timing of their administration. The results were promising but inconclusive. Pre-emptive analgesia through the administration of local anesthetic as a regional field block perioperatively has been investi- 
gated in multiple surgical disciplines. The meta-analysis by Ong et al. [1] evaluated the use of multiple pre-emptive analgesic methods; the use of preemptive local anesthetics was found to provide a clinically useful reduction in supplemental analgesic consumption and a prolonged time to first rescue analgesic request. However, similar to the results of our study, pain intensity measures were not found to be significantly reduced by pre-emptive analgesia with local anesthetics $[1,20]$. Despite this, an important advantage of pre-emptive analgesia that the Ong group discussed is an economic one. Reducing postoperative analgesic use via the adoption of pre-emptive analgesia techniques significantly lowers total hospitalization costs [1]. As spinal surgery pushes further into the ambulatory settings, measures like local anesthetic pre-emptive analgesia will become ever more important to make this transition feasible.

The use of a local anesthetic infiltration prior to surgical incision is a common practice by many, and was a routine in our practice prior to the initiation of this PI project. The addition of epinephrine to the injectate has the dual increased benefit of prolonging the action of the local anesthetic and helping with hemostasis of the incised skin. Although the preoperative neuropathic pain symptoms are often relieved in patients following a successful decompression, a large proportion of the postoperative pain is incisional in nature due to the invasive dissection that is required. Inadequate pain control has been linked with many negative outcomes both clinically and economically, including poorer patient satisfaction scores, increased length of hospital stay, slower time to mobilization, and slower functional recovery [17]. In this study, we investigated the use of LB infiltration at wound closure as an adjuvant for improving postoperative pain control in patients undergoing a single-level TLIF, which regardless of technique (i.e., open versus minimally-invasive) is a very invasive operation that requires significant musculoskeletal dissection and retraction. As seen in our results and those previously reported, patient reported pain scores following major spinal surgery tend not to be correlated to pain medication consumption, suggesting the reliance on pain scores alone to assess pain control may be misleading [1].

Both cohorts were similar in the demographics analysis, including preoperative average pain scores and average preoperative ODI. The results of our study support many of the findings demonstrated in the limited number of studies that exist about pre-emptive analgesia with LB in spine surgery. The most obvious and statistically significant difference between the two cohorts was a decrease in the use of morphine equivalents of intravenous narcotics from the DOS to POD 3 in the patients who received LB. This finding is clinically important because it is the need for intravenously dosed "rescue" opioids that is often a barrier to discharge, as opposed to the total pain medication consumption. This result is also demonstrated in the Puffer et al. [4] and Kim et al. [17] studies analyzing the use of LB in spine surgery. However, compared to the Kim group's study, our study cohorts all had surgeries performed by a single surgeon, with standardized surgical technique and a protocoled perioperative care plan, thus isolating LB infiltration in a standard dose and method of distribution during closure as the single significant difference between the two cohorts. In addition, the interchangeable use of lidocaine or bupivacaine infiltration prior to incision (medication and dosage was based on surgeon preference) in the Kim et al. [17] study could be a confounding variable which we eliminated with the use of a standardized dose of $10 \mathrm{~mL} 0.5 \%$ bupivacaine with epinephrine administered intradermally and subcutaneously local to the planned incision site. Likewise, and potentially more biasing in that study, was the lack of a standardized dose and location of infiltration of LB following completion of the TLIF procedure. In the current study, all patients in the LB cohort received a set dose in the supra-fascial, subdermal location, divided equally between the left and right side of the exposure. Despite key methodological differences between the Kim et al. [17] study and ours, the two are synergistic in that the most significant finding in each was that administration of LB during closure results in a substantial decrease in the need for intravenous narcotic pain medications in the acute perioperative period. The total amount (oral+intravenous) of narcotic consumption in the LB cohort was on average 12-16 mg morphine equivalents lower than the control cohort on DOS to POD 2. This difference stabilized to nearly identical morphine equivalents on POD 3 between the two cohorts. These are similar findings as in the Surdam et al. [6] study in which LB was compared to a femoral nerve block (FNB) in patients undergoing unilateral total knee arthroplasty. They found a statistically significant decrease in the amount of total narcotic pain medication consumed on POD 1 in the LB cohort compared to the FNB cohort (LB, 3.9 versus FNB, 9.1) [6]. In addition, 
despite not achieving statistical significance, the trend they found was towards a lower total narcotic consumption on POD 2 in the LB cohort (LB, 1.5 versus FNB, 4.3) and then a similar total narcotic consumption between the two cohorts by POD 3 (LB, 0 versus FNB, 1.7) [6]. This equilibration of total narcotic use on POD 3 in the Surdam et al. [6] study and our PI project may be attributed to LB's 72-hour duration of action. The trend towards lower total narcotic consumption with LB use and a statistically significant reduction in intravenous narcotic use allows for a quicker transition to a stable oral medication regimen, quicker mobilization postoperatively (evaluated in Surdam et al. [6] study), and a reduced length of stay. Supporting this improved transition concept was the fact that, the LB cohort spent an average of 15.8 hours less on PCA than the control group; however, due to the limited numbers followed under our PI project, we were unable to demonstrate statistical significance $(p=0.1506)$.

We hypothesized that average length of stay would be decreased as well in the LB cohort, unfortunately such a difference was not demonstrated (LB cohort, 4.8 days; control group, 5.1 days) despite similar studies showing a statistically significant reduction in length of stay between the two cohorts $[6,17]$. The similar length of stay in our study may be better explained by the practice setting of this study. The study was performed in an active US military medical center located in Germany where length of stay is more protocol-based than a civilian practice in the United States. Future studies in different practice settings with different preset preoperative expectations for length of stay would be helpful to further investigate whether LB can lead to a reduced hospital stay as well as reduced perioperative consumption of narcotic pain medications. We did see a decrease in the total number of patients requiring an APS consult in the LB cohort. This is an economically promising result since pre-emptive analgesia may be a means for employing fewer hospital resources to achieve adequate postoperative pain control, especially when these resources are sparse or absent in certain settings (i.e., ambulatory or same-day surgery centers). Consistent with other studies investigating the utility of LB, we were not able to show any significant differences in VAS pain scores at any time point $[4,20]$.

Based on the results of this study and prior reports, LB use may help mitigate postoperative narcotic use and its inherent drawbacks. The adverse effects of narcotics are well known; however, adoption of pain as a "fifth vital sign" has generated a dilemma for physicians, who are now under increased scrutiny to optimize pain control, especially in the hospital setting, while at the same time avoid over use of opiates [21]. As a result, pharmaceutical companies have employed different tactics to combat the addiction potential of opiate pain medications, such as longer-acting formulations; however, evidence supporting their reduced addiction potential and efficacy over short-acting opioids is inconclusive [22]. Complications with opioid medication use in chronic pain patients are particularly challenging, hence the interest in alternative modalities in perioperative pain management. One interesting phenomenon of long-term opioid use is opioidinduced hyperalgesia $(\mathrm{OIH})$, a syndrome of increased sensitivity to painful stimuli, in which the dose-effect curve is inverted, such that more narcotic medication results in lower pain control [22]. Buprenorphine and gabapentin are being investigated for the management of this complication that arises with prolonged opiate use [23]. The use of agents such as gabapentin to manage complications like $\mathrm{OIH}$ in chronic pain patients segues into the concept of using multi-modal therapy to pre-emptively reduce opiate medication use postoperatively. Different medications and techniques aimed at reducing opioid pain medication consumption following surgery include: neuraxial opioids as intrathecal or epidural administration, nonsteroidal anti-inflammatory drugs, $\mathrm{N}$-methyl D-aspartate receptor antagonists (including ketamine, methadone, dextromethorphan, and magnesium), alpha-2 receptor agonists (clonidine and dexmedetomidine), gabapentin and pregabalin, and intravenous lidocaine [24]. With so many options and conflicting data available to support each of their use, it is challenging to implement a pain regimen for patients undergoing surgery that is optimally effective and cost-conscientious. While the literature may be ambiguous regarding specifics on which modality is best, one factor that has been shown repeatedly to be superior to opiate-based mono-therapies is the use of multimodal analgesia for postoperative pain control [25]. In the context of this PI project, we have demonstrated that the infiltration of LB into the superficial wound at the end of spinal procedures is one such modality that is effective and leads to demonstrable improvements in process of care measures. Based on this work, LB infiltration following major (and minor) spine surgery at our institution has become a standard practice [4].

Although we were able to show statistically significant 
differences between the two cohorts, and in turn confirm several of our hypotheses regarding LB use, future prospective well-powered studies are needed to investigate and confirm differences and benefits attributable to this approach. The key question that remains unanswered by our work and that already published, is the differential benefit of cheaper local anesthetics, like plain regular bupivacaine, versus the more expensive LB option studied herein. Likewise, what location (i.e., supra- or subfascial) and what dose is ideal. Multi-center prospective trials are being planned to address these remaining unanswered questions, as well as to confirm or refute the benefits that have been demonstrated in previous level 3 evidence or lower work.

Subcutaneous infiltration of LB during wound closure in patients undergoing a single-level TLIF is effective in reducing postoperative intravenous narcotic use, and tends to result in less time spent on PCA and fewer APS consultations. Further studies are necessary to investigate this and other methods of pre-emptive analgesia in spine surgery to determine multi-modal approaches to pain control that are both effective and economical.

\section{Conclusions}

Subcutaneous infiltration of LB during wound closure in patients undergoing a single-level TLIF is effective in reducing postoperative intravenous narcotic use, and tends to result in less time spent on PCA and fewer APS consultations. Further studies are necessary to investigate this and other methods of pre-emptive analgesia in spine surgery to determine multi-modal approaches to pain control that are both effective and economical.

\section{Conflict of Interest}

No potential conflict of interest relevant to this article was reported.

\section{References}

1. Ong CK, Lirk P, Seymour RA, Jenkins BJ. The efficacy of preemptive analgesia for acute postoperative pain management: a meta-analysis. Anesth Analg 2005;100:757-73

2. Tong YC, Kaye AD, Urman RD. Liposomal bupivacaine and clinical outcomes. Best Pract Res Clin An- aesthesiol 2014;28:15-27.

3. Grieff AN, Ghobrial GM, Jallo J. Use of liposomal bupivacaine in the postoperative management of posterior spinal decompression. J Neurosurg Spine 2016;25:88-93.

4. Puffer RC, Tou K, Winkel RE, Bydon M, Currier B, Freedman BA. Liposomal bupivacaine incisional injection in single-level lumbar spine surgery. Spine J 2016;16:1305-8.

5. Bramlett K, Onel E, Viscusi ER, Jones K. A randomized, double-blind, dose-ranging study comparing wound infiltration of DepoFoam bupivacaine, an extended-release liposomal bupivacaine, to bupivacaine $\mathrm{HCl}$ for postsurgical analgesia in total knee arthroplasty. Knee 2012;19:530-6.

6. Surdam JW, Licini DJ, Baynes NT, Arce BR. The use of exparel (liposomal bupivacaine) to manage postoperative pain in unilateral total knee arthroplasty patients. J Arthroplasty 2015;30:325-9.

7. Kalogera E, Bakkum-Gamez JN, Weaver AL, et al. Abdominal incision injection of liposomal bupivacaine and opioid use after laparotomy for gynecologic malignancies. Obstet Gynecol 2016;128:1009-17.

8. Miranda SG, Liu Y, Morrison SD, et al. Improved healthcare economic outcomes after liposomal bupivacaine administration in first-stage breast reconstruction. J Plast Reconstr Aesthet Surg 2016;69:1456-7.

9. Abdelsattar JM, Boughey JC, Fahy AS, et al. Comparative study of liposomal bupivacaine versus paravertebral block for pain control following mastectomy with immediate tissue expander reconstruction. Ann Surg Oncol 2016;23:465-70.

10. Jackson SM, Whitlark JD. Pain control with liposomal bupivacaine after thoracoscopies/thoracotomies. Ann Thorac Surg 2015;100:2414-5.

11. Knight RB, Walker PW, Keegan KA, et al. A randomized controlled trial for pain control in laparoscopic urologic surgery: $0.25 \%$ bupivacaine versus long-acting liposomal bupivacaine. J Endourol 2015;29:101924.

12. Butz DR, Shenaq DS, Rundell VL, et al. Postoperative pain and length of stay lowered by use of exparel in immediate, implant-based breast reconstruction. Plast Reconstr Surg Glob Open 2015;3:e391.

13. Robbins J, Green CL, Parekh SG. Liposomal bupivacaine in forefoot surgery. Foot Ankle Int 2015;36:5037. 
14. Beck DE, Margolin DA, Babin SF, Russo CT. Benefits of a multimodal regimen for postsurgical pain management in colorectal surgery. Ochsner J 2015;15:408-12.

15. Hutchinson HL. Local infiltration of liposome bupivacaine in orthopedic trauma patients: case-based reviews. Am J Orthop (Belle Mead NJ) 2014;43(10 Suppl):S13-6.

16. Schmidt WK, Patou G, Joshi GP. Evaluating therapeutic benefit in postsurgical analgesia requires global assessment: an example from liposome bupivacaine in hemorrhoidectomy. Hosp Pract (1995) 2012;40:160-5.

17. Kim J, Burke SM, Kryzanski JT, et al. The role of liposomal bupivacaine in reduction of postoperative pain after transforaminal lumbar interbody fusion: a clinical study. World Neurosurg 2016;91:460-7.

18. Woolf CJ. Evidence for a central component of postinjury pain hypersensitivity. Nature 1983;306:686-8.

19. Woolf CJ, Chong MS. Preemptive analgesia: treating postoperative pain by preventing the establishment of central sensitization. Anesth Analg 1993;77:36279.
20. Moiniche S, Kehlet H, Dahl JB. A qualitative and quantitative systematic review of preemptive analgesia for postoperative pain relief: the role of timing of analgesia. Anesthesiology 2002;96:725-41.

21. Ling W, Mooney L, Hillhouse M. Prescription opioid abuse, pain and addiction: clinical issues and implications. Drug Alcohol Rev 2011;30:300-5.

22. Chou R, Clark E, Helfand M. Comparative efficacy and safety of long-acting oral opioids for chronic non-cancer pain: a systematic review. J Pain Symptom Manage 2003;26:1026-48.

23. Compton P, Kehoe P, Sinha K, Torrington MA, Ling W. Gabapentin improves cold-pressor pain responses in methadone-maintained patients. Drug Alcohol Depend 2010;109:213-9.

24. Dunn LK, Durieux ME, Nemergut EC. Non-opioid analgesics: novel approaches to perioperative analgesia for major spine surgery. Best Pract Res Clin Anaesthesiol 2016;30:79-89.

25. Mathiesen O, Dahl B, Thomsen BA, et al. A comprehensive multimodal pain treatment reduces opioid consumption after multilevel spine surgery. Eur Spine J 2013;22:2089-96. 